\title{
ACTIVITIES IN AEROELASTICITY AT NASA LANGLEY RESEARCH CENTER
}

\author{
Boyd Perry, III and Thomas E. Noll
}

\author{
NASA Langley Research Center \\ Hampton, Virginia
}

USA

\begin{abstract}
This paper presents the results of recently-completed research and presents status reports of current research being performed within the Aeroelasticity Branch of the NASA Langley Research Center. Within the paper this research is classified as experimental, analytical, and theoretical aeroelastic research. The paper also describes the Langley Transonic Dynamics Tunnel, its features, capabilities, a new open-architecture data acquisition system, ongoing facility modifications, and the subsequent calibration of the facility.
\end{abstract}

\section{INTRODUCTION}

Aeroelasticity at the NASA Langley Research Center has a rich and varied heritage, including both theory and experiment. Over sixty years ago, at Langley, Theodore Theodorsen performed his groundbreaking theoretical work in understanding the mechanism of flutter (Theodorsen, 1935). A quarter century later, at Langley, the premier aeroelasticity testing facility in the world, the Langley Transonic Dynamics Tunnel (TDT), became operational and has now served the aeroelasticity community for close to four decades. The Langley "family tree" in aeroelasticity, from the 1930's to the present, includes other such notables as Garrick, Watkins, Runyan, Woolston, Donely, Houbolt, Cunningham, Reed, and Yates.

The current work in aeroelasticity being performed at NASALangley continues the tradition of making relevant contributions to the state-of-the-art. The purposes of this paper are two-fold: (1) to present the results of recent experimental, analytical, and theoretical activities in fixed-wing and rotary-wing aeroelasticity at NASA-Langley; and (2) to briefly describe the heavy gas conversion project currently underway at the TDT and the associated calibration of the tunnel.

\section{TRANSONIC DYNAMICS TUNNEL}

The TDT, shown in Figure 1, is a unique "national" facility dedicated to identifying, understanding, and solving relevant aeroelastic problems. It is the best suited wind tunnel in the world for flutter testing large, full-span, aeroelastically-scaled models at transonic speeds (Staff of the Aeroelasticity Branch, 1969).

\section{Tunnel Characteristics}

The TDT is a closed-circuit, continuous-flow, variablepressure, wind tunnel with a 16-foot square test section. The tunnel uses either air or a heavy gas as the test medium and can operate at stagnation pressures from near vacuum to atmospheric, has a Mach number range from near zero to about 1.2 , and is capable of maximum Reynolds numbers of about 3 million per foot in air to about 10 million per foot in heavy gas.

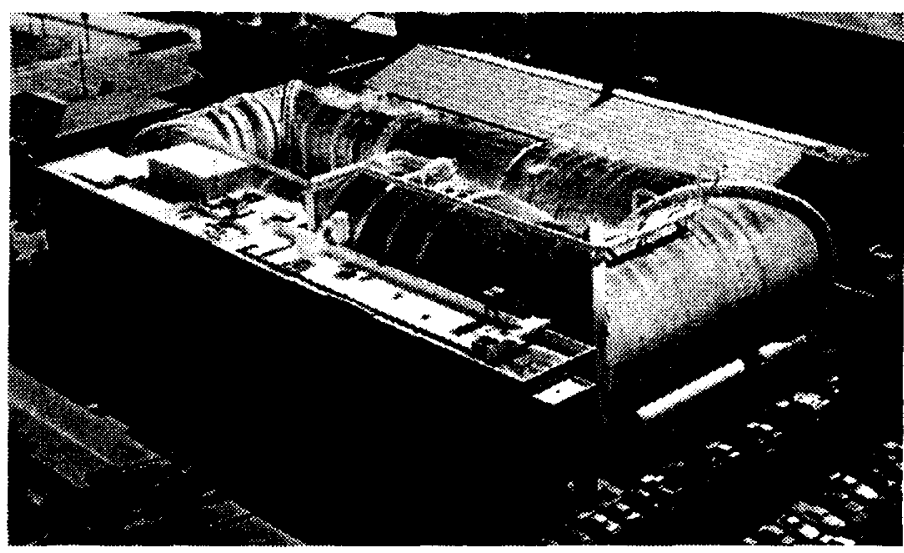

Figure 1: NASA Langley Transonic Dynamics Tunnel 
The TDT is specially configured for flutter testing, with excellent model visibility from the control room and a rapid tunnel shutdown capability for model safety. Model mount systems include two sidewall turntables for semispan models, a variety of stings for full-span models, a cable-mount system for "flying" models, a rotorcraft testbed for rotor blade loads research, and a floor turntable for launch vehicle ground-wind loads studies. The TDT also offers an airstream oscillation system for gust studies and supporting systems for active controls testing. Testing in heavy gas has important advantages over testing in air including improved model to full-scale similitude, higher Reynolds numbers, and reduced tunnel power requirements.

\section{Facility Modification}

As stated above, the TDT relies on a heavy gas test medium. Currently the TDT is undergoing a major facility modification to alter the heavy gas processing equipment and to replace the previous heavy gas with an environmentally friendly heavy gas. The chemical name of the new heavy gas is 1,1,1,2tetrafluoroethane, or, more conveniently, R-134a. The facility modifications are expected to be completed in the summer of 1997 , followed, first, by an extensive system "shakedown" and, next, with a thorough calibration of the flow characteristics of the wind tunnel. The TDT will become fully operational with the new gas during the winter of 1998.

\section{Calibration of Tunnel}

The objectives of the TDT calibration are to determine the operating envelope for the new test medium, to determine appropriate techniques for quantifying TDT flow parameters, and to quantify test section flow uniformity and flow quality in air and in $\mathrm{R}-134 \mathrm{a}$.

Primary flow parameters will be calibrated using test section centerline and sidewall pressure measurements. These measurements will be obtained with a centerline tube instrumented to measure static pressure in the settling chamber and through the test section and with instrumentation mounted along the test section walls, ceiling, and floor to measure static pressures. Total and static pressure and total temperature will also be measured at discrete locations in the settling chamber and test section. These latter measurements in the settling chamber and test section will be used to calculate the primary flow conditions for the facility. It is anticipated that empirical corrections to the flow properties will be made based on the measurements obtained with the centerline tube and sidewall static pressure measurements.

Measurements using a flow survey rake and boundary layer rakes will also be obtained during the TDT calibration to assess test section flow uniformity and quality. The flow survey rake will be instrumented to measure spatial variations in flow angularity, Mach number, and turbulence as functions of tunnel speed. The boundary layer rakes will show boundary layer variations along the tunnel walls, ceiling, and floor. Several boundary layer rakes which can be mounted at different streamwise locations within the test section will be used to determine boundary layer characteristics as a function of test section station.

\section{Data Acquisition System}

Testing of aeroelastic models in the TDT requires real-time acquisition and display of measured static and dynamic experimental data, and complex, on-line analyses of dynamic data. The TDT open-architecture dynamic data acquisition system (TDT-DAS) is a state-of-the-art system which meets these requirements.

The TDT-DAS hardware is comprised of three subsystems, each switch-connectable to a subset of four NEFF "front ends." Each NEFF provides signal conditioning, filtering, and sampleand-hold analog-to-digital conversion for 64 channels for a total capability of 256 channels. Two of the subsystems are comprised of a Motorola $\mathbf{8 8 0 0 0}$ series computer and the third is comprised of a Digital Equipment Corporation Alpha series computer. These computers perform the basic data acquisition, archiving to disk, and continuous buffering of data to a fiber-optic data ring. Connected to the fiber-optic ring is an SGI Challenge $\mathrm{L}$ computer which provides both on-line frequency analysis, and post-point time- and frequency-domain data analysis and controllerperformance evaluation.

All computers and terminals are connected, via networks, to workstations at the TDT or to workstations at remote sites, providing a distributed real-time data display capability. One of the first successful demonstrations of this capability was accomplished with a real-time link to the Aeronautical and Maritime Research Laboratory in Melbourne, Australia. For this demonstration data was acquired from two strain gages mounted on a flexible wing set up in a laboratory at the TDT. Output from the strain gages was acquired by the TDT-DAS at a sampling rate of 1000 samples per second per channel. Using a reflective memory network the data was then transferred to an SGI Challenge $\mathrm{L}$ computer (at Langley) for conversion to engineering units and display. TDT-DAS personnel then opened a connection to Australia (an IP address of the "target" Macintosh in Australia had been supplied), providing real-time data access ten thousand miles away.

\section{EXPERIMENTAL AEROELASTIC RESEARCH}

Historically, the TDT has been used to perform flight envelope flutter clearance tests for new commercial and military flight vehicles and to conduct research investigations that address aeroelastic issues plaguing both fixed-wing and rotarywing vehicles. This section of the paper presents the results of some recent (1993 to 1996) research-related wind-tunnel test demonstrations.

\section{Piezoelectric Aeroelastic Response Tailoring Investigation (PARTI)}

The objective of the PARTI program (McGowan, 1996) was to demonstrate the ability of strain-actuated adaptive wings to 
control aeroelastic response and to prevent flutter. For this demonstration a five-foot long, high-aspect-ratio semispan flutter model was fabricated for testing in the TDT. The model consisted of an exterior fiberglass shell to provide the proper aerodynamic contouring and an interior composite plate to serve as the main load carrying structure. A sketch of the major components of the PARTI wing are shown in Figure 2. Piezoelectric actuator patches were distributed on both the upper and lower surfaces of the composite plate inboard of the 60 percent wing span. Due to the ply orientation of the material used in the composite plate and the wing sweep, the piezoelectric actuator patches were able to affect both the bending and the torsional responses of the model. Ten strain gages and four accelerometers were available as feedback sensors and for monitoring the model response during the tests. As shown in the figure, the model was also equipped with a trailing-edge aerodynamic control surface and with an automatic flutter-stopper.

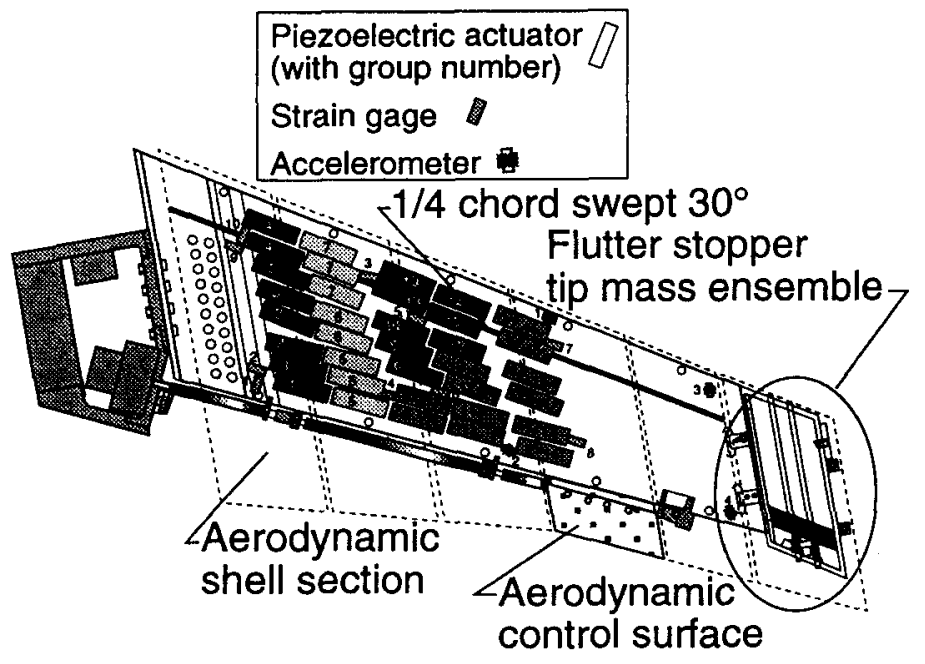

\section{Figure 2: Sketch of the Major Components of the} PARTI Wind-Tunnel Model

For this program two wind-tunnel test entries were performed. The first entry was used to measure the model's openloop response including its flutter characteristics and to determine time-history information for each important piezoelectric actuator group. The second entry was used to assess and demonstrate the capability of piezoelectric actuators to suppress flutter and to reduce aeroelastic response caused by turbulence. Control laws were designed using experimentally determined state-space models and actuator transfer functions. Twenty-eight control laws, designed using various techniques and different actuator groups and sensor signals, were tested. The complexity of the control laws varied from single-input/singleoutput to multi-input/multi-output controllers having five sensors and nine actuator groups. The most successful control law was found to increase the flutter dynamic pressure by 12 percent and to reduce the power spectral density of peak response due to tunnel turbulence (Figure 3) at speeds below flutter by $75 \%$.

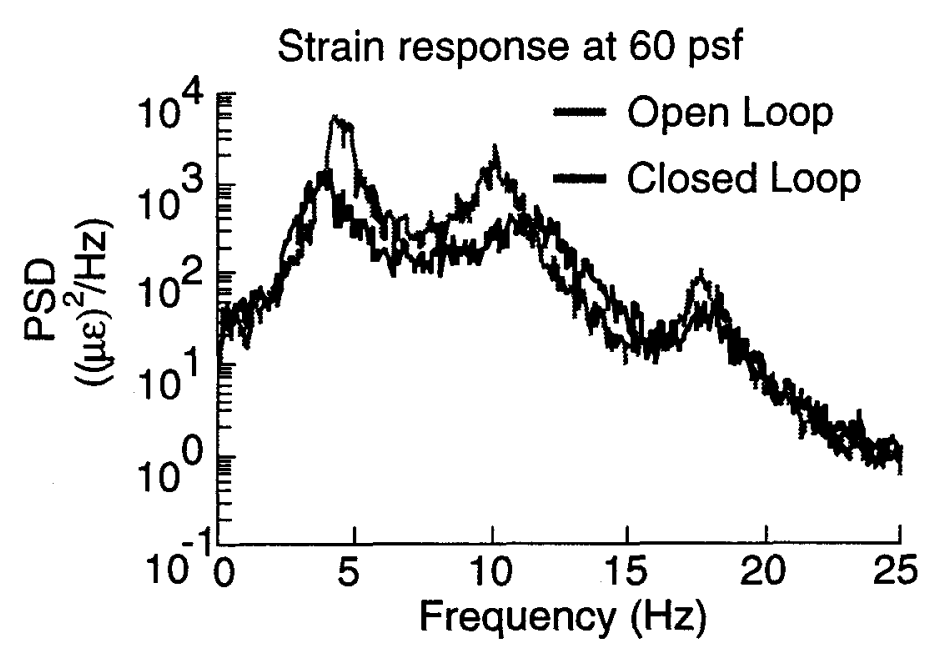

Figure 3: PARTI Turbulence Response Results. Mach Number $=0.43 ;$ Dynamic Pressure $=60$ psf

Future plans in this area will be focused on combining the capability of neural network controllers with smart material actuators for aeroelastic applications. The neural network methodology offers the opportunity to adapt to changing flight conditions or dynamics and to reconfigure the sensors and actuators following failures.

\section{Benchmark Active Controls Technology (BACT)}

NASA Langley's Benchmark Models Program (BMP) was initiated in the late 1980 s to study the physics of aeroelastic phenomena and to acquire an experimental aerodynamic database for code validation. The BACT program was part of the BMP with an emphasis on active controls. The objectives of the BACT program were to: obtain high quality data to validate CFD and computational-aeroelasticity codes; to verify the accuracy of aeroservoelastic analysis tools; and to provide a testbed for evaluating innovative control methodologies. The BACT model (Figure 4) was a rigid, rectangular, pressure-instrumented wing with an NACA 0012 airfoil. It had three control surfaces (a trailing-edge control surface and upper- and lower-surface spoilers) powered by hydraulic actuators. To study aeroelastic instabilities the model was attached to a flexible mount system which provided the two flexible degrees of freedom required for classical flutter. To obtain overall static and dynamic loads the flexible mount system was not employed and the model was "rigidly" mounted to a force balance.

Initially, open-loop tests were conducted to define the model's aerodynamic characteristics, including the flutter boundary across the transonic Mach number range. An extensive database of over 3000 data sets including steady and unsteady control surface effects was obtained (Scott, 1997). Closed-loop tests were performed next to evaluate various nonadaptive flutter suppression control law concepts. Spoiler surfaces alone and the trailing-edge control surface alone were each effective in preventing flutter, but, when used in combination, the spoiler and 
trailing-edge surface provided superior flutter suppression capability. This superior performance was typified by much more rapid suppression of flutter motion and "capture" of the model at the conclusion of a "system on - system off - system on" sequence.

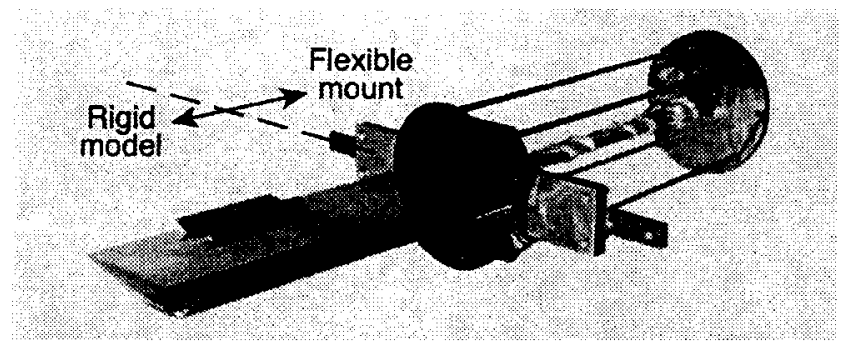

\section{Figure 4: BACT Wind-Tunnel Model and Flexible Mount System}

Tests were also performed to evaluate a variety of semiadaptive control algorithms for flutter suppression and gust load alleviation. Figure 5 presents some of the closed-loop results for three different concepts. The solid line is the open-loop flutter boundary and the circles correspond to the points where the various control concepts were tested. The three concepts evaluated included: a Generalized Predictive Control (GPC) algorithm that employed an analytical representation of the plant to predict future model responses and to select control surface commands; an Inverse Control (IC) method that used a linear neural network and experimental data to define the plant inverse; and a Neural Predictive Control (NPC) algorithm (Lichtenwalner, 1996). All three systems were successful in suppressing flutter. A gust load alleviation system was also successfully demonstrated; the system was able to reduce model acceleration response by 80 percent in the presence of flow oscillations caused by the TDT airstream oscillator system.

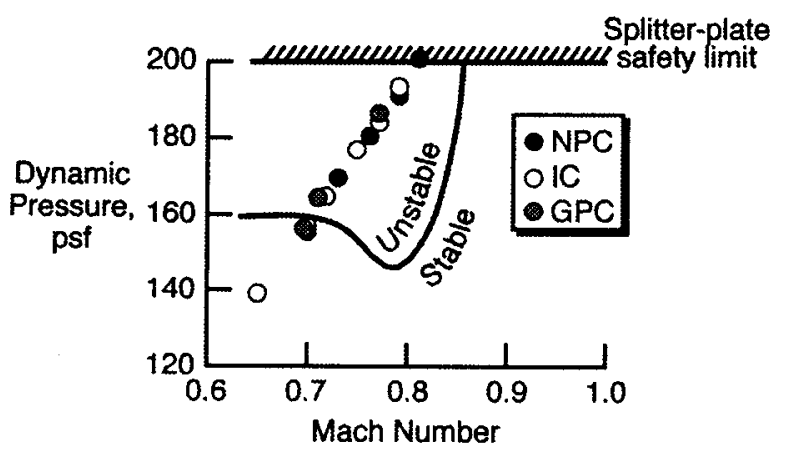

Figure 5: BACT Semi-Adaptive-Control Flutter Suppression Results

\section{Actively Controlled Response of Buffet Affected Tails (ACROBAT)}

Buffet is an aeroelastic phenomenon which plagues high performance aircraft, especially those with twin vertical tails. For aircraft of this type at high angles of attack, vortices emanating from wing/fuselage leading edge extensions burst, immersing the vertical tails in their wake. The resulting buffet loads on the vertical tails are a concern from a fatigue point of view.

The objective of the ACROBAT (Moses, 1997) program was to demonstrate, through tests in the TDT, the feasibility of using active controls to suppress vertical tail buffeting. Three new flexible vertical tails were fabricated for use on an existing, 1/6scale, sting-mounted F-18 model (Figure 6). Each tail was equipped with a different control device: a rudder surface; a tip vane configuration containing a slotted cylinder or an embedded slotted cylinder; and piezoelectric actuators. All three flexible tails were instrumented with a root strain gage aligned to measure bending moment and with two tip accelerometers located near the leading and trailing edges.

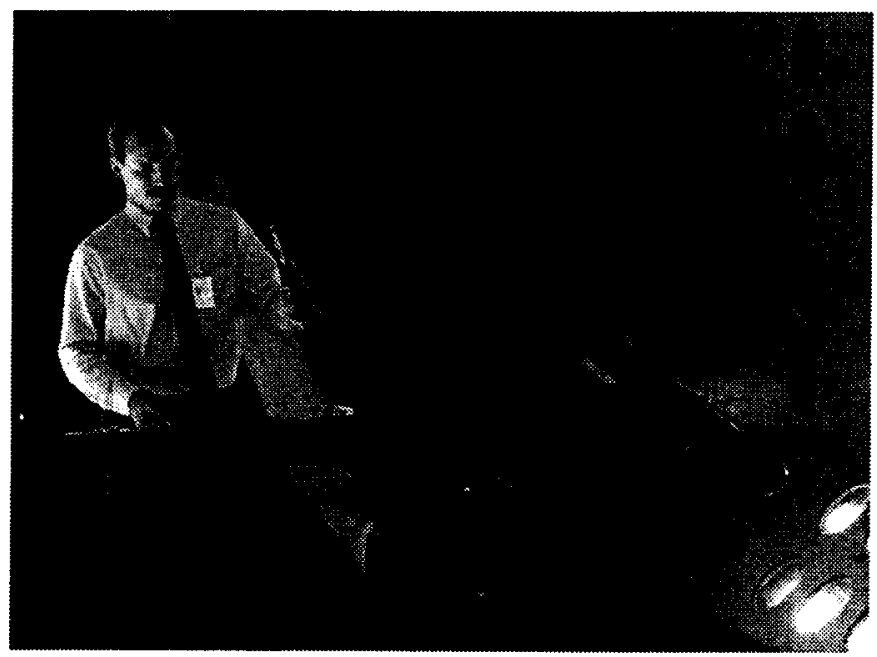

Figure 6: ACROBAT Wind-Tunnel Model Mounted in the TDT

Wind-tunnel tests were performed with the model angle of attack varying from 20 to 37 degrees. Data were measured for several cases: open loop (no actuator commands); actuator commanded by a linear sweep; actuator commanded by constant frequency sinusoidal motion; and closed loop (control laws were operating). It was determined that the control systems using either the rudder or the piezoelectric actuators were best for suppressing the buffeting. With a single-input/single-output, constant gain control law, the peak of the power spectral density function of the root bending moment at the frequency of the first bending mode was reduced by as much as $60 \%$ (Figure 7) for certain angles of attack, using gains well below the physical limits of the actuator being investigated. At angles of attack up to about 30 degrees, both devices were nearly equally effective in alleviating buffeting, however at higher angles of attack, the piezoelectric devices had superior performance. 


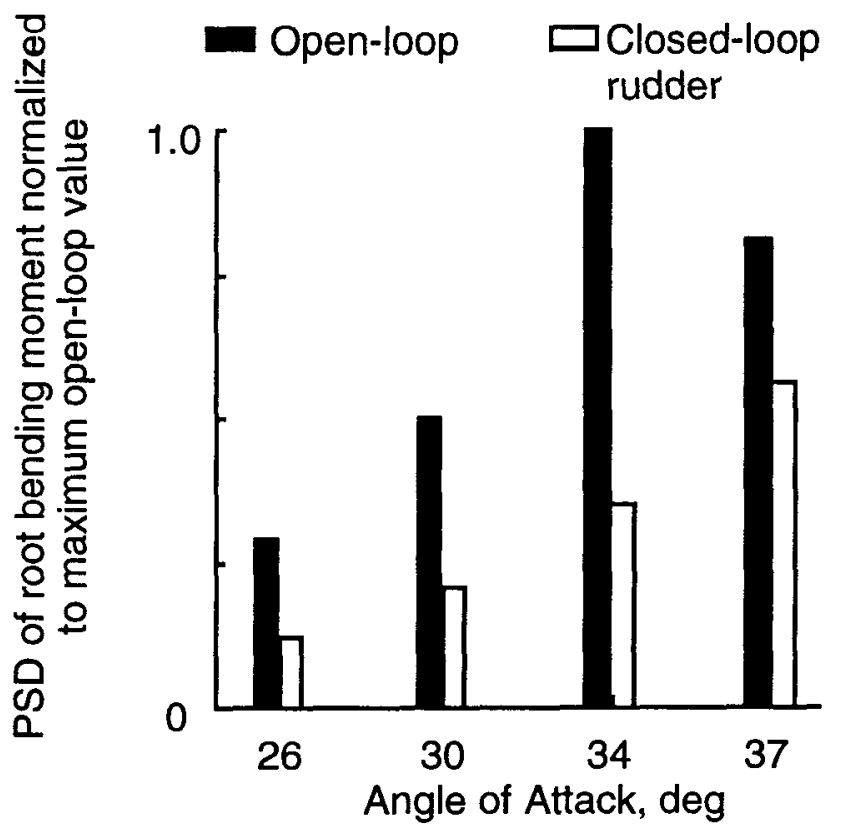

a) Rudder results.

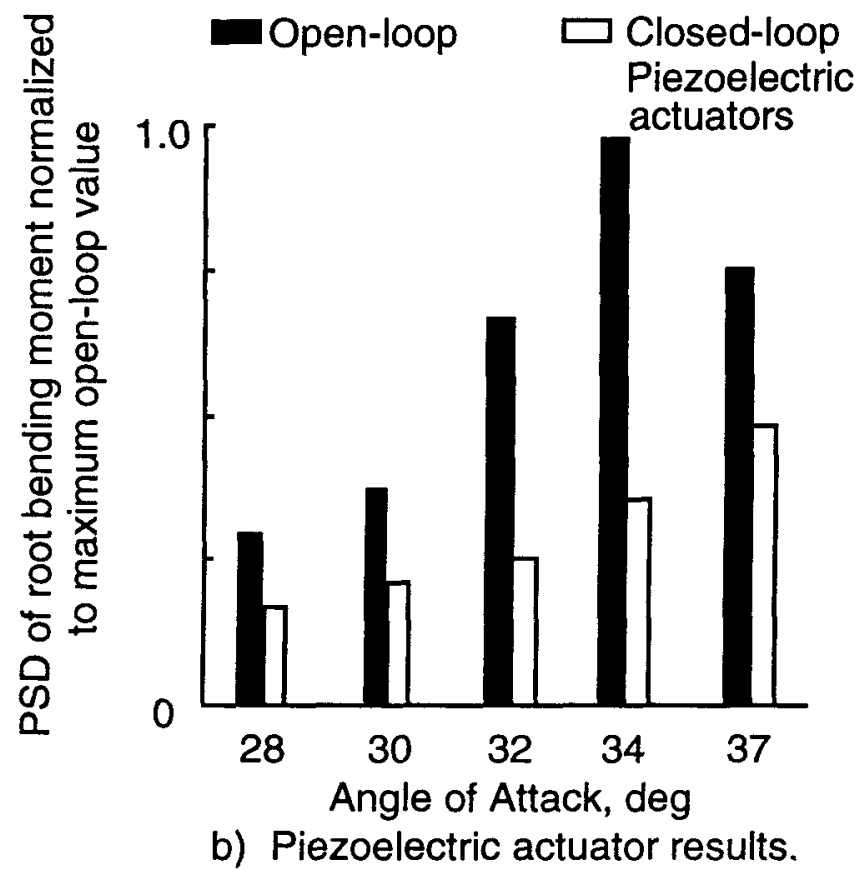

Figure 7: Normalized Power Spectral Density of Root Bending Moment at the Frequency of the First Bending Mode for Open-Loop and Closed-Loop

Systems. Mach Number $=0.10$; Dynamic Pressure $=14$ psf

Through the auspices of TTCP (The Technical Cooperation Program), researchers from several international government laboratories will participate in a piezoelectric actuator buffet alleviation ground test experiment in Melbourne, Australia using a full-scale F-18 aircraft. Taking advantage of this investigation, NASA plans to fabricate new vertical tails with embedded piezoelectric actuators that match the full-scale ground test configuration and to perform tests in the TDT to investigate scaling issues related to smart materials.

\section{Wing and Rotor Aeroelastic Testing System (WRATS)}

An aggressive wind-tunnel test program was initiated to address tiltrotor aeroelastic research issues as identified by: 1) the NASA Short-Haul Civil Tiltrotor (SH-CT) Program; 2) U.S. rotorcraft industry with regard to the development of marketable tiltrotor technologies; and 3) the U.S. Army with regard to the development of high-speed rotorcraft capabilities. A key issue to improving marketability of current tiltrotor systems is to reduce noise and weight and to improve aerodynamic performance. Such reductions and improvements generally result in an associated detrimental impact on the loads, vibration, and aeroelastic stability of the vehicle. The objective of the WRATS (Nixon, 1997) program is to evaluate the ability of an active system to control fixed-system vibrations associated with the multiple modes of tiltrotor flight vehicles. The WRATS tiltrotor testbed is a semispan model developed from a V-22 1/5-scale aeroelastic tiltrotor model. A photograph of the WRATS model mounted in the TDT is shown in Figure 8.

An active control system using three high-frequency hydraulic actuators to tilt the swashplate and an active flaperon is employed on the WRATS model. This active control system is known as MAVSS (Multipoint Adaptive Vibration Suppression System) and was developed by Bell Helicopter Textron, Inc. The actuators are driven by a signal produced by the active system at frequencies up to $50 \mathrm{~Hz}$. The active system operates in the following manner: it obtains feedback signals from response sensors (beam, chord, and torsion strain gage bridges); it quantifies model vibration levels in an objective function; it identifies the system using a series of test signals; and it computes and then applies commands to either the active swashplate or the active flaperon to lower the objective function. If the vibration level rises above a given threshold, the controller will automatically reactivate itself.

Figure 9 contains results from the TDT test and illustrates the success of the active system in controlling vibratory loads in three wing modes simultaneously. Each set of three vertical bars grouped together indicates the three-per-rev (3P) wing beam, chord, and torsion loads. For each of the four airspeeds there are a set of bars shown with the active system both off and on. The plot shows a trend of increasing baseline $3 \mathrm{P}$ vibration level in all three wing modes with airspeed, but, more importantly, also shows significant reductions $(89 \%$ to $99 \%$ ) in all $3 \mathrm{P}$ vibratory wing loads at each airspeed. Although not shown on the figure, the swashplate and flaperon motions required to accomplish these reductions are within acceptable limits. 


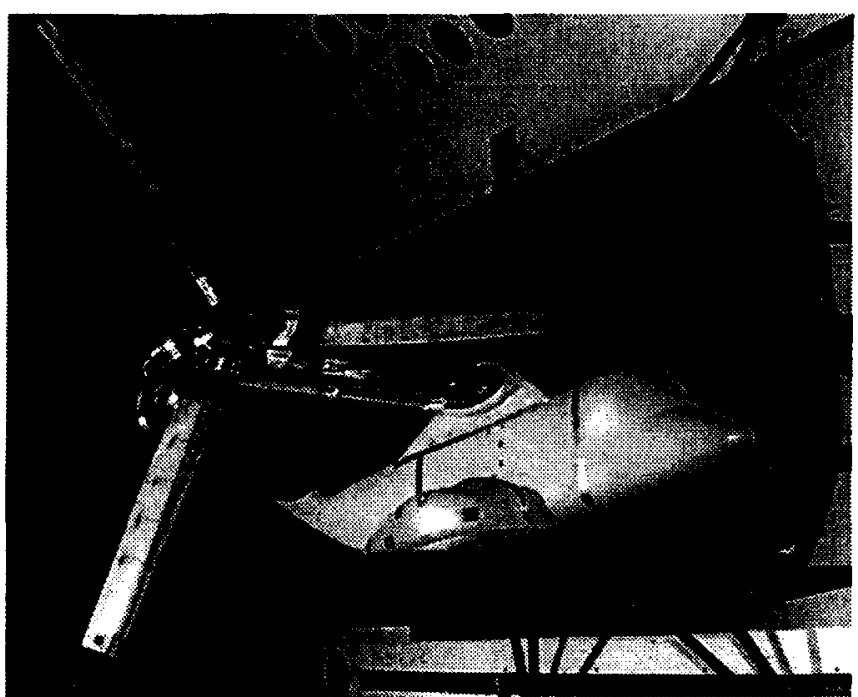

Figure 8: WRATS Wind-Tunnel Model Installed in the TDT

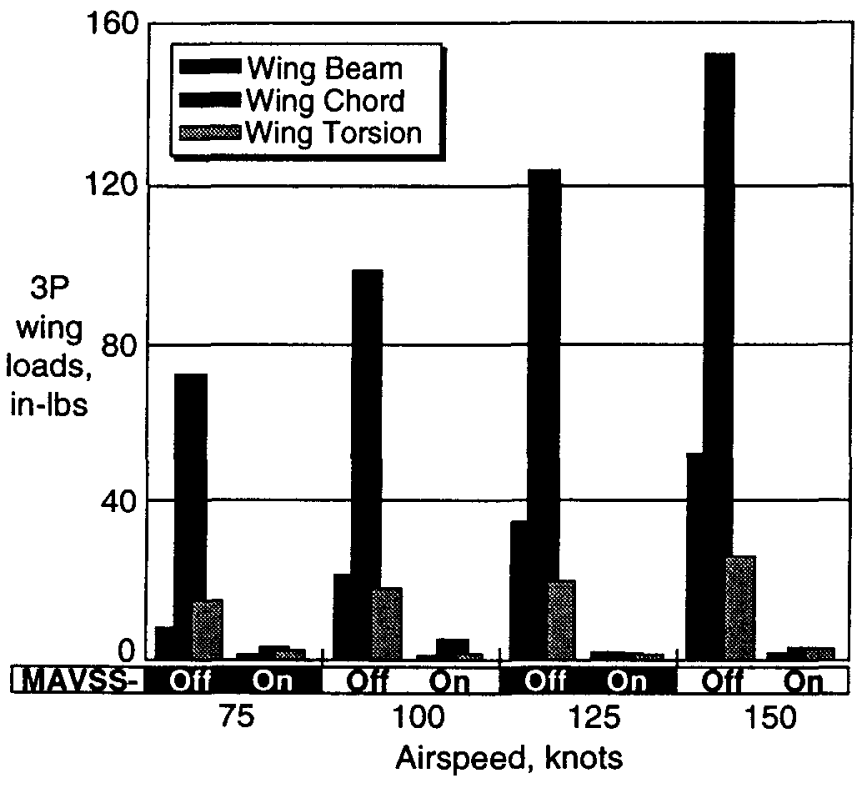

Figure 9: Effect of MAVSS on Loads as a Function of Airspeed

\section{High Speed Research (HSR) Aeroelasticity Program}

The objective of the aeroelasticity task of the HSR program is to provide validated analyses, design tools, and demonstrated technology readiness to accurately predict and solve the aeroelastic problems of a high-speed civil transport (HSCT). An important element of the aeroelasticity task is a wind-tunnel models program in which a series of models of increasing complexity is fabricated and tested in the TDT to study the basic flow physics and flutter mechanisms of an HSCT configuration. Three models are planned to be tested: a rigid semispan model (RSM); a flexible semispan model (FSM); and an actively controlled, flexible, full-span, cable-mount model (FFM). Below, a brief overview of the RSM and FSM tests are provided. Since a model like the FFM is very expensive and risky to test, an available flexible model of a 1970's supersonic transport design (referred to as the Active Controls Testbed) was tested in the TDT to develop testing procedures and data reduction/evaluation tools needed for the FFM wind-tunnel model program. Some conclusions resulting from this test are also reviewed below.

Rigid Semispan Model. The RSM (Figure 10) is an extensively instrumented, stiff, semispan wing/fuselage model. The objective of this series of tests was to acquire baseline pressure and loads data free from significant aeroelastic deformations. The wing is a graphite-epoxy composite structure with removable engine nacelles. It has a control surface located near the inboard trailing edge of the wing which can be statically deflected and/or oscillated.

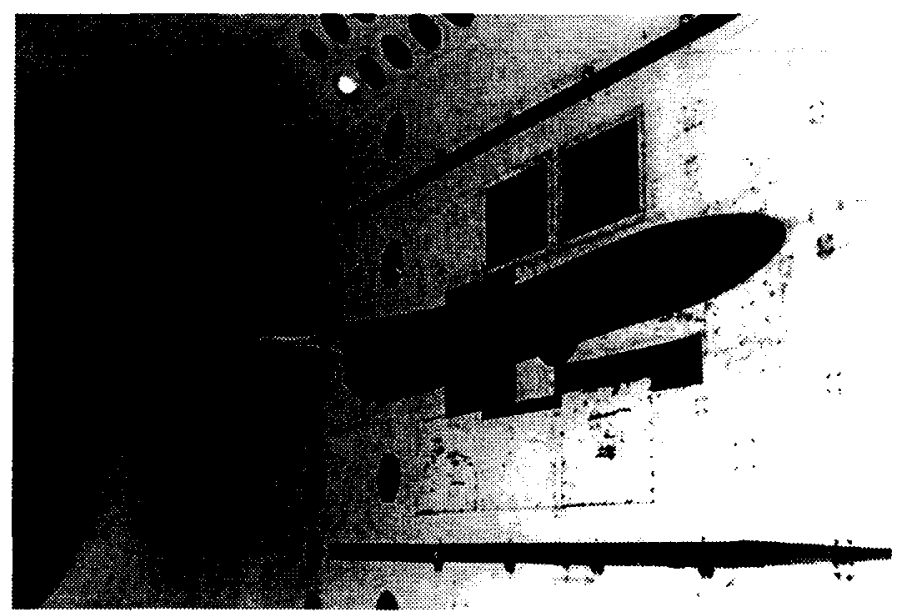

Figure 10: HSR Rigid Semispan Model Installed in the TDT

Wing pressure instrumentation consisted of 135 in-situ unsteady pressure transducers distributed along four chords on the upper and lower surface. The wing is mounted to a force balance which is attached to the TDT sidewall turntable. The fuselage is instrumented at seven fuselage stations with a total of 120 steady pressure orifices and is mounted to the turntable independent of the balance. This mounting arrangement allows the RSM wing and fuselage to be pitched to the same angle-ofattack while only the aerodynamic loads on the wing are measured by the balance. A video based deflection measurement system was also used to measure wing-tip deflections during testing. An extensive database consisting of steady wing and fuselage pressure, aerodynamic load, and wing-tip deflection data were obtained over a large range of freestream Mach numbers $(0.70$ to 1.15), angles-of-attack $\left(-2^{\circ}\right.$ to $\left.+8^{\circ}\right)$, control surface deflection angles $\left(-4^{\circ}\right.$ to $\left.+4^{\circ}\right)$, and control surface oscillations (amplitudes up to $5^{\circ}$ at frequencies up to $10 \mathrm{~Hz}$ ) with and without engine nacelles. In addition, approximately 6200 reflective targets were 
attached to the upper and lower surfaces of the RSM and photogrammetric data were obtained to define the exact model shape for CFD modeling and analyses.

Flexible Semispan Model. The FSM had the same geometrical scale as the RSM, but had the aeroelastic characteristics of an HSCT design. Like the RSM, the FSM was also highly instrumented. The instrumentation included 135 unsteady pressure transducers on the wing at four chord locations, 80 steady pressure transducers on the fuselage, 14 accelerometers in the wing, and strain gages (to monitor loads at critical areas of the wing). The model was mounted on a fivecomponent instrumentation balance to measure wing-only loads. A large database consisting of steady and unsteady aerodynamic pressures and loads and forced and unforced dynamic response data (with sine-dwell and sine-sweep excitations to the trailing edge control surfaces) was established for various angles of attack and control surface deflections at Mach numbers from 0.7-1.15 at dynamic pressures of 100 and 150 psf. Dynamic measurements were also made at conditions near or at flutter and at transonic conditions of high model response. Figure 11 provides a comparison of lift curve slope between the RSM and the FSM.

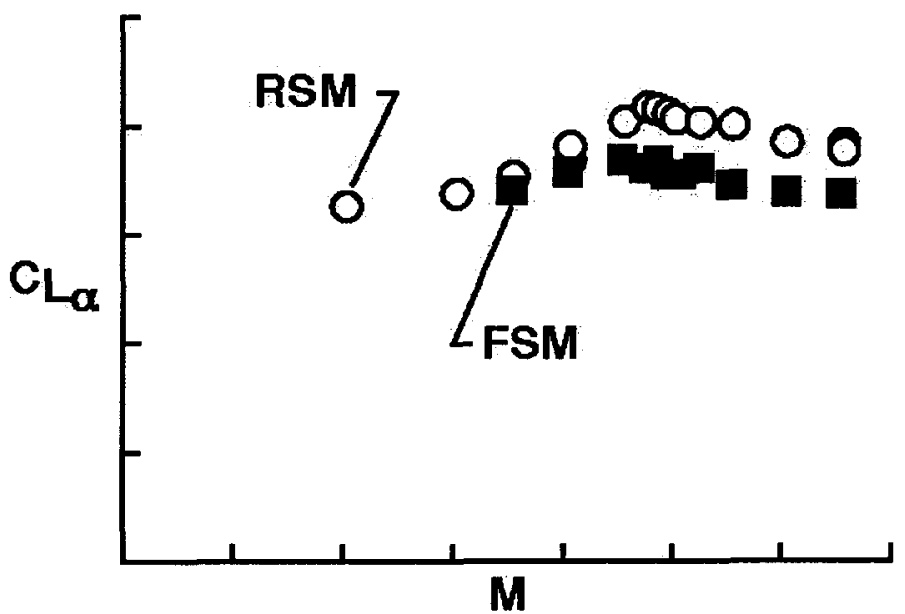

\section{Figure 11: Lift Curve Slope Versus Mach Number for HSR Rigid and Flexible Semispan Models}

The flutter, loads, and steady and unsteady pressure measurements made for the RSM and FSM, as well as the model ground test measurements, represents an unprecedented aeroelastic models data base. The data provide the foundation necessary to assess the validity and applicability of various computational fluid dynamics (CFD) and aeroelastic codes that will be used in the HSR program and in the eventual design of an HSCT aircraft.

Active Controls Testbed. Since the current HSR program calls for active-controls testing of a full-span, cablemounted aeroelastic model in the TDT, an available full-span, dynamically scaled, model (Figure 12) of an SST was used as a testbed to assess the testing procedures associated with large, flexible, cable-mounted models and to evaluate available data analysis and reduction tools. The model had hydraulically operated inboard and outboard trailing edge control surfaces and a stiff horizontal stabilizer with a geared elevator. The wings were of a stressed skin design of fiberglass skins over balsa wood and fiberglass ribs and spars. Instrumentation, which included strain gages, accelerometers, and rate gyros, were used to monitor for flutter, to measure model response, and to provide feedback signals for stability augmentation systems. This model was tested both on a sting and on the TDT's cable mount system to obtain its flutter and cable-mount stability characteristics, and to investigate closed-loop stability augmentation systems.

The test results were mixed. On one hand the test demonstrated that a stability augmentation system (which added damping to the pitch and plunge "flying" modes of this large flexible model on the cable mount system) can be designed, implemented and successfully tested. In this regard the test was very successful. However, on the other hand, in testing a modified stability augmentation control law, this test revealed some deficiencies in testing practices and revealed a conceptual error in an important on-line analysis capability, which, in combination, led to the destruction of the model. Unknowingly, the loop was closed on an unstable control law which caused the model to enter a cable-mount instability from which recovery was impossible. As a result of this test and the subsequent improvements in testing practices and corrections to software, the risk of losing the FFM under similar circumstances has been greatly reduced.

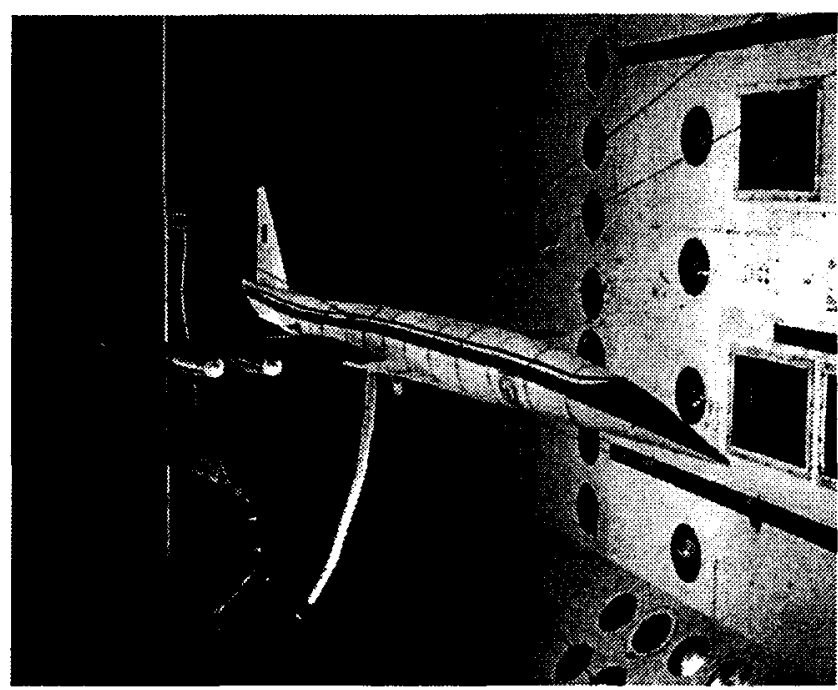

Figure 12: Active Controls Testbed Installed on the TDT Cable Mount System 


\section{ANALYTICAL AEROELASTIC RESEARCH}

This section of the paper highlights recent studies aimed at enhancing and validating a computational aeroelasticity code based on transonic small disturbance theory and rotorcraft analysis codes which predict the structural dynamic and aeroelastic characteristics of helicopter and tiltrotor vehicles.

\section{Computational Aeroelasticity Method for Predicting Transonic Instabilities}

The CAP-TSD (Computational Aeroelasticity ProgramTransonic Small Disturbance) potential equation code was recently extended (Edwards, 1993) to include a viscous-inviscid interactive coupling method referred to as Interactive Boundary Layer Modeling (IBLM) to better predict unsteady transonic flows involving separation and reattachment. The IBLM is regarded as a simulation of two dynamic systems, the outer inviscid flow (freestream region) and the inner viscous flow (boundary layer region), whose coupling requires active control elements to minimize the coupling error between the two systems. The motivation for this enhancement was to obtain the capability to predict transonic shock-induced flow separation instabilities and response phenomena. A recent paper (Edwards, 1996) summarizes the results of applying the enhanced code (referred to as CAP-TSDV) to the solution of several challenging aeroelastic problems. Analyses were conducted to predict: the buffet onset boundary of an NACA 0012 airfoil; the shock-induced oscillations of an $18 \%$ circular arc airfoil; and the transonic flutter boundary both for a thin wing and for a typical business jet wing. All the predictions agreed well with experiment.

The CAP-TSDV flutter predictions for the relatively thick wing of a business jet are shown in Figure 13 in the form of flutter speed index versus Mach number. For purposes of comparison, these predictions are shown with others computed by Gibbons (1996). The open circle symbols are the experimental data; the open diamond symbols represent the small-amplitude flutter boundary predicted by the inviscid TSD solution and are seen to depart from the experimental results with increasing Mach number; the open square symbols and upright and inverted triangle symbols represent the small-amplitude flutter boundary computed by computational methods of increasing sophistication incorporating viscous effects. The solid square symbol represents a large-amplitude limit cycle oscillation computed by the CAPTSDV code at Mach number 0.888. The large-amplitude LCO computed by CAP-TSDV is in good agreement (in terms of amplitude and frequency of motion) with model behavior observed during the test and captured on video tape.

Present plans are to continue applying the CAP-TSDV code to a variety of transonic aeroelastic phenomena with an emphasis on control surface buzz and limit cycle oscillations. Upon validation, our intent is for the code to be used during the design phase of new flight vehicles and as a means of identifying and assessing "fixes" following unexpected transonic aeroelastic instabilities being encountered in flight.

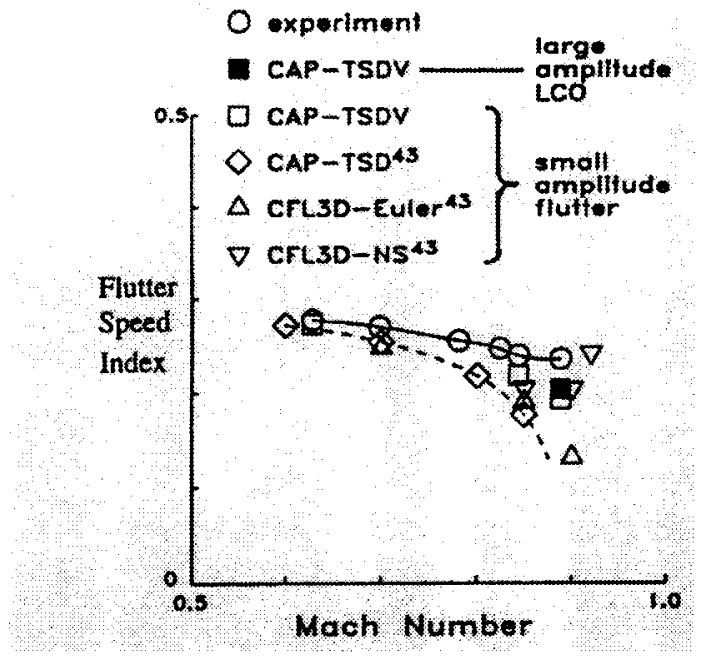

a) Flutter speed index

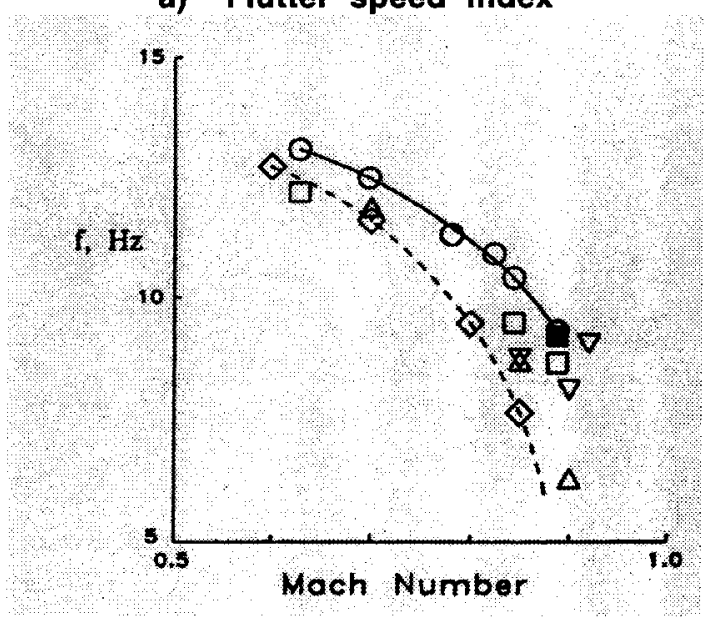

b) Flutter frequency

Figure 13: Comparison Between Experimental and Calculated Flutter Boundaries for a Flutter Model of a Typical Business Jet

\section{Design Methodology for Low Vibration Helicopter Rotors}

Helicopter vibration is a significant problem that can reduce aircraft efficiency and productivity because of its effect on crew members, aircraft components, and passengers. One effective way of reducing vibration is through structural tailoring of rotor blades during the design phase. To accomplish a reasonable and cost effective design in the most efficient manner, a validated analysis is needed to allow the blade designer to determine the values of parameters that produce the lowest vibration levels and to incorporate these values in the blade final design. A study which involves performing analyses and conducting tests in the TDT is underway to establish an analytical capability that can be routinely utilized as a tool in the design of low vibration helicopter rotors. 
The second generation of the Comprehensive Analytical Model of Rotorcraft Aerodynamics and Dynamics code (CAMRAD II) (Johnson, 1980) was selected for the analytical portion of this study. CAMRAD II calculates rotorcraft performance, loads, vibration, and stability. Presently, an existing set of TDT data is being used to compare with CAMRAD II predictions of rotor performance and loads. The analysis closely predicted the rotor performance, the overall phase character of the loads, as well as the magnitudes of the loads.

During the next step in the study, a set of rotor blade parameters known to have significant effects on vibratory loads will be selected and CAMRAD II analyses will be performed to determine the sensitivity of rotor and fixed-system loads to perturbations in each of the parameters. Model rotor blades will then be fabricated, tested in the TDT to experimentally determine the effect of the selected parameters on rotor loads, and correlated with the CAMRAD II calculations to verify the capability of the code as a tool for use in the design of low vibration rotors.

\section{Proprotor Aeroelastic Stability Analysis (PASTA)}

The PASTA code was originally developed in the mid $1980 \mathrm{~s}$ to support the TDT flutter tests of a $1 / 5$-scale aeroelastic model of the Bell/Boeing V-22 tiltrotor aircraft and then-emerging NASALangley tiltrotor aeroelastic research programs. The code was based on research conducted a decade earlier (Kvaternik, 1973). To support current industry wide tiltrotor initiatives, several recent enhancements to the PASTA code have been implemented and verified. These new features include: (1) a five-degree-offreedom drive system math model; (2) improved treatment of airframe support springs for representing spring-supported windtunnel models; (3) ability to account for engine gyroscopic effects associated with pitching and yawing of the pylon; and (4) approximate treatment of proprotor steady-state thrust. Present plans are to continue enhancing the code to include quasi-steady wing aerodynamic matrices from s-plane approximations of unsteady generalized aerodynamics and to implement active control strategies for vibration reduction and stability augmentation.

\section{Tiltrotor Leads and Aeroelastic Stability}

Aeroelastic predictions of tiltrotor loads and stability have been improved through enhancements to the University of Maryland Advanced Rotorcraft Code (UMARC) (Nixon, 1993). Enhancements to the code began at LaRC in 1992, but continue to be added and refined today. These recent additions include: 1) a fully anisotropic beam element with implicit shear deformation for modeling both rotor blades and wings; 2) a gimbal hub modeling capability which works in conjunction with the bearingless, hingeless, and articulated rotor systems already available in UMARC; 3) trim analysis options for the full range of pylon conversion operation; 4) an expanded stability analysis which accounts for the fully-coupled wing/pylon/blade motions; and 5) a drive-train model for improved analysis of tiltrotor lag mode dynamics.
The new UMARC code introduces unique analysis capabilities which have proved useful in both government and industry research efforts. A NASA-Langley study showed the potential for expanding the tiltrotor stability boundaries (associated with pylon whirlflutter at high speeds) by 100 knots through use of advanced composite materials which provide bending-twist coupling in the blades (Figure 14). In addition,

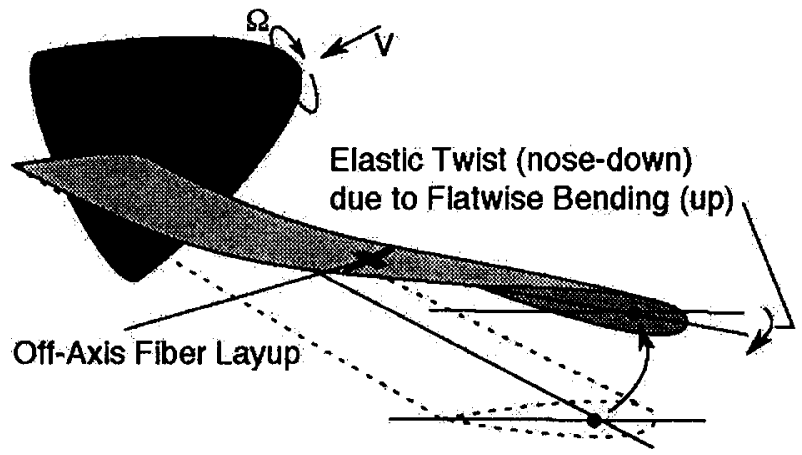

a) Bending-twist-coupled blade

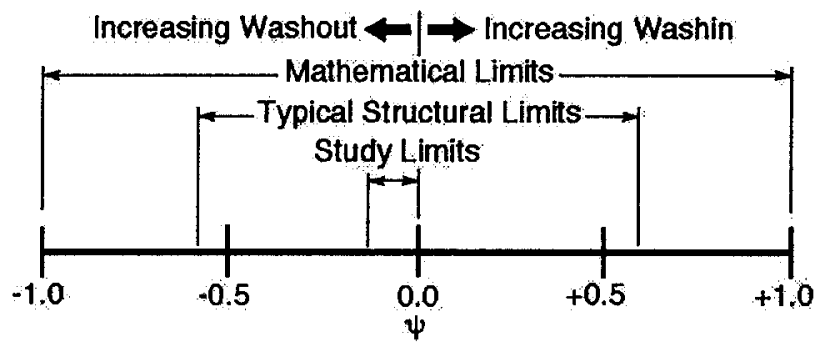

b) Bending-twist-coupling parameter

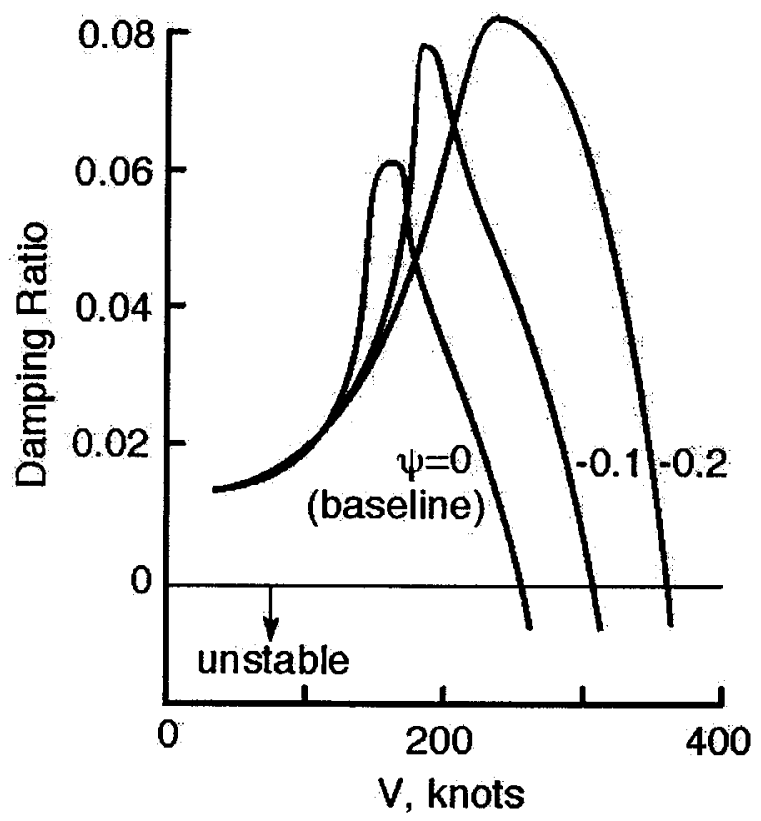

c) Stability of wing beam-bending mode

Figure 14: Bending-Twist-Coupled Rotor Blade Analysis Results from the Improved UMARC Code 
the analysis enhancements have been adopted by Sikorsky Helicopter engineers who continue to refine the Sikorsky Variable Diameter Tiltrotor design based in part on loads and stability predictions of the code.

The UMARC tiltrotor analysis code is currently being refined through an exhaustive validation effort associated with recent loads and stability testing of WRATS tiltrotor testbed which was discussed earlier in this paper.

\section{THEORETICAL AEROELASTIC RESEARCH}

This section of the paper describes theoretical activities in aeroelasticity underway at NASA Langley. These studies include: the application of Volterra nonlinear discrete-time filter theory to nonlinear discrete-time models and an aeroelastic analysis of helicopter rotor blades incorporating embedded piezoelectric fiber composite twist actuation systems.

\section{Discrete-Time Volterra Theory}

Current research in the application of linear and nonlinear digital filter techniques to CFD codes has led to: 1) a new understanding of linear unsteady aerodynamic responses, including the classical Wagner, Kussner, Theodorsen, and Sears functions; and 2) a computationally efficient method for computing nonlinear steady and unsteady aerodynamic responses.

Traditionally viewed as an abstract and impractical function, the current research has identified the importance and usefulness of a mathematically-valid and numerically-accurate aerodynamic impulse response function. Previous work (Silva, 1993) was an early exploration into this development, but only recently was the fundamental importance of the aerodynamic impulse response function recognized. Once the aerodynamic impulse response function is known, convolution of it with any input will yield the response of the linear system to that input. Examples: convolution of the impulse response with a step angle-of-attack input yields the indicial response (generalized Wagner's function); convolution of the impulse response with a sinusoidal angle-of-attack input yields the simple harmonic motion response, or the Doublet-Lattice result (generalized Theodorsen's function); convolution with step and sinusoidal gust inputs yields gust responses (generalized Kussner's and Sears' functions).

Figure 15 contains two comparisons of "actual" versus convoluted responses of a three-dimensional rectangular wing with an NACA 0012 airfoil at Mach number 0.9 due to an arbitrary plunging motion using the CAP-TSD code. The top comparison uses the linear equations in CAP-TSD and the results, as expected, are identical with and cannot be distinguished from each other in the figure. In the bottom comparison, a nonlinear case, the discrete-time Volterra theory of nonlinear systems provides a process similar in efficiency to the linear case but with a mathematically-rigorous capability to account for nonlinearities. The comparison of the actual nonlinear solution versus the Volterra first-order ("convolved") nonlinear solution shows some small, almost indistinguishable, differences, but the two solutions are, in general, in very close agreement, illustrating the power of the digital convolution technique.

Because the linear and nonlinear responses are computed only once, in using the digital convolution technique linear and nonlinear responses of the system to arbitrary inputs can be generated on a workstation, eliminating the need to reprocess information via the CFD code, resulting in significant time and computational cost savings. In addition to the CAP-TSD examples presented here, this method has been successfully applied to the viscous Burger's equation and to the CFL3D Navier-Stokes flow solver.

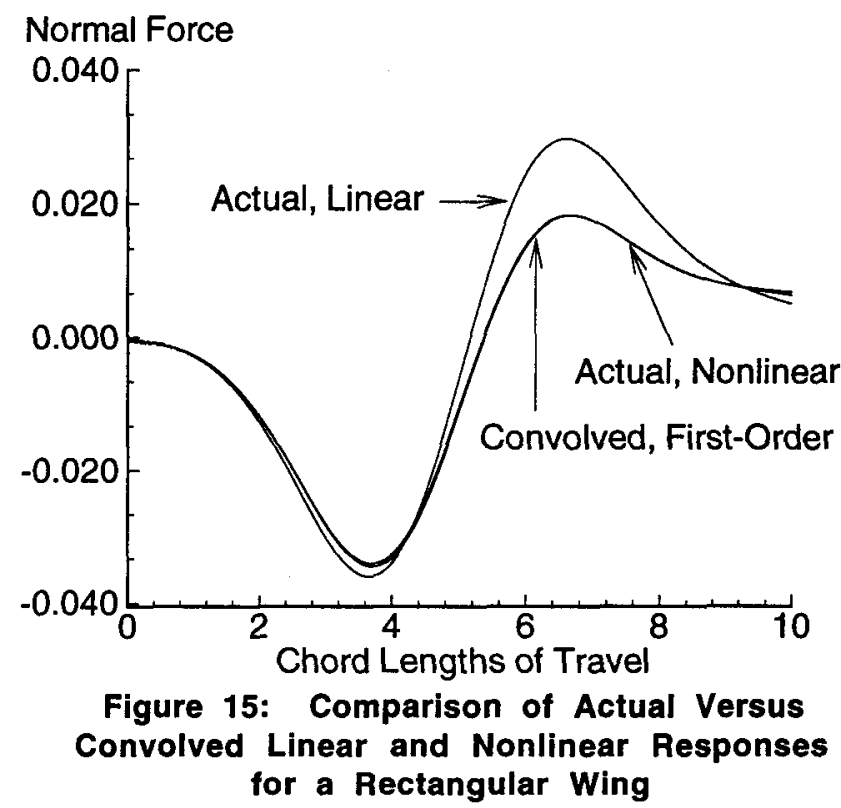

\section{Aeroelastic Modeling of Rotor Blades with Embedded Piezoelectric Fibers}

Blade twisting using embedded or bonded piezoceramic actuators has been proposed as a superior means of performing individual blade control on helicopters. Generating the magnitudes of elastic twisting necessary for such an approach to be successful has thus far proven to be difficult or impossible with conventional through-plane poling techniques and piezoceramic materials. The recent development of advanced piezoelectric actuation schemes using anisotropic piezoelectric actuators could make individual blade control using active blade twisting feasible. 

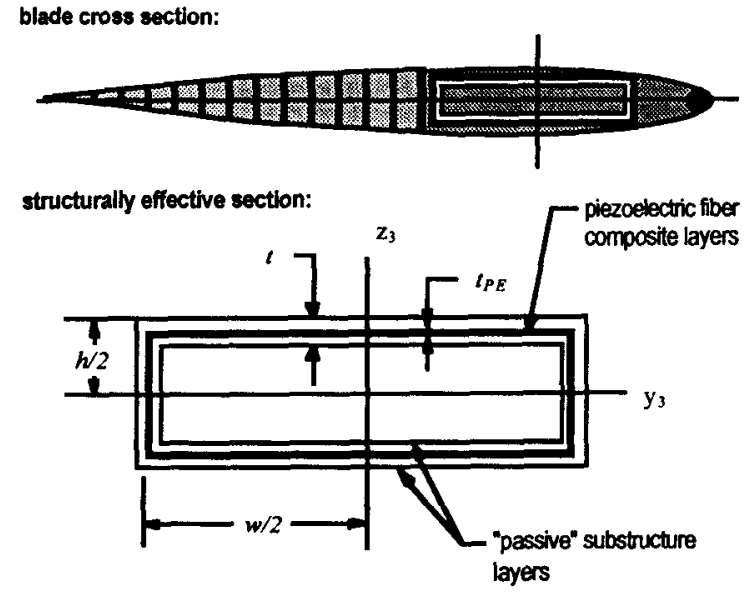

a) Idealized rectangular, thin-walled, closed-
section piezoelectric blade structure
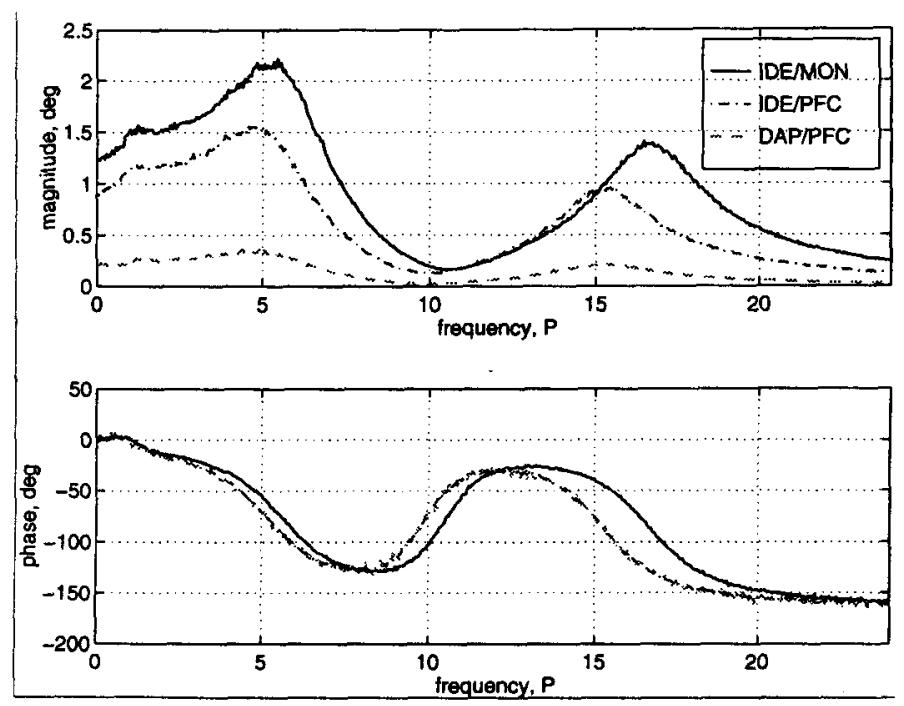

b) Results

\section{Figure 16: Comparison of Piezoelectric Twist Actuation for $1 \mathrm{~g}$ Hovering Flight}

Recently, (Wilkie, 1996) the capability of advanced anisotropic piezoelectric actuators to produce dynamic twisting on helicopter rotor blades was examined. An idealized rotor blade structural geometry, consisting of a thin walled rectangular box spar with embedded piezoelectric actuator laminae, was assumed for the purposes of these studies (Figure 16a). Both free-strain anisotropy and structural anisotropy were permitted in these piezoelectric laminae to make their analysis applicable to as wide a range of actuation configurations as possible. This enabled the actuation capabilities of monolithic piezoceramics, piezoelectric fiber composites (PFC), and interdigitated-electrode-poled (IDE) piezoceramics to be represented.
The relative capabilities of three twist actuation schemes, each employed on a realistically scaled rotor blade structure, were numerically examined. Each of these configurations represented a special case of anisotropic piezoelectric actuation. These special cases were: 1) anisotropic piezoelectric free-strain with isotropic in-plane stiffness, corresponding to a composite rotor blade structure containing interdigitated-electrode-poled, monolithic piezoceramic plies (IDE/MON); 2) anisotropic free-strain and anisotropic stiffness, representing interdigitated-electrode-poled, piezoelectric fiber composite plies (IDE/PFC); and 3) isotropic free-strain with anisotropic stiffness, representing conventional, though-plane poled piezoelectric fiber composite plies (DAP/PFC). Hovering flight conditions were used for all cases.

A comparison of the magnitudes and phase of blade twist produced for these three configurations as a function of the applied electric field frequency is shown in Figure 16b. The effect of the large free-strain anisotropies present in the IDE schemes on the magnitude of elastic twist is readily apparent. Both of the IDE poling cases exhibit generally four to five times the twist actuation magnitudes of the conventionally poled configuration. In addition, the magnitudes shown for the IDE cases in the $2 \mathrm{P}$ to $5 \mathrm{P}$ frequency range, i.e., on the order of one to two degrees of amplitude, are generally regarded as being sufficient for an effective active twist vibration reduction scheme.

These results demonstrate that current piezoceramic materials can potentially produce useful amounts of dynamic blade twisting on helicopter rotor blades if used with an interdigitated electrode poling scheme. The aeroelastic analysis developed for this study has also proven to be a useful tool for the conceptual design of active twist rotor blade structures, and is presently being used to study the dynamic stall behavior of active twist helicopter rotor blades, and to design model-scale active twist rotor blades suitable for testing in the TDT.

\section{CLOSING REMARKS}

This paper has presented an overview of recent and current work in fixed-wing and rotary-wing aeroelasticity within the Aeroelasticity Branch at the NASA-Langley Research Center. This paper contains both accomplishments and status reports of the wide variety of experimental, analytical, and theoretical research being conducted here. At the heart of the experimental work is the Langley Transonic Dynamics Tunnel, which, at this writing, is in the midst of a major facility modification. The modification and associated tunnel calibration are expected to be completed and the TDT is scheduled to be back "on-line" by early 1998. In the years to come NASA will continue to conduct research in aeroelasticity in an effort to fully understand and predict the aeroelastic behavior of aerospace vehicles so that future designs can fully exploit these technology advances.

\section{ACKNOWLEDGMENT}

The authors wish to acknowledge the work of the research staff of the Aeroelasticity Branch at NASA Langley Research 
Center whose research is being reviewed in this paper. In addition the authors wish to acknowledge our research partners from the U.S. aviation industry, government labs, and academia for their contributions to our program.

\section{REFERENCES}

Edwards, J., "Transonic Shock Oscillations Calculated with a New Interactive Boundary Layer Coupling Method," AIAA Paper 93-0777, January 1993.

Edwards, J., "Transonic Shock Oscillations and Wing Flutter Calculated with an Interactive Boundary Layer Method," Proceedings of the EUROMECH-Colloquium 349, Simulation of Fluid-Structure Interaction in Aeronautics, Gottingen, Germany, September 1996.

Gibbons, M., "Aeroelastic Calculations Using CFD for a Typical Business Jet Model," NASA CR 4753, September 1996.

Johnson, W.: "A Comprehensive Analytical Model of Rotorcraft Aerodynamics and Dynamics." NASA TM-81182, 1980.

Kvaternik, R., "Studies in Tilt-Rotor VTOL Aircraft Aeroelasticity," PhD Dissertation from Case Western Reserve University, June 1973.

Lichtenwalner, P., Little, G., and Scott, R., "Adaptive Neural Control of Aeroelastic Response," Proceedings of the 37 th AIAA/ASME/ASCE/AHS/ASC Structures, Structural Dynamics, and Materials Conference, Salt Lake City, UT, April 1996.

McGowan, A., Heeg, J., and Lake, R., "Results from WindTunnel Testing from the Piezoelectric Aeroelastic Response Tailoring Investigation," Proceedings of the 37 th AIAA/ASME/ASCE/AHS/ASC Structures, Structural Dynamics, and Materials Conference, Salt Lake City, UT, April 1996.

Moses, R. "Active Vertical Tail Buffeting Alleviation on a Twin-Tail Fighter Configuration in a Wind Tunnel," Proceedings of the CEAS International Forum on Aeroelasticity and Structural Dynamics, Rome, Italy, June 1997.

Nixon, M. W., "Aeroelastic Response and Stability of Tiltrotors with Elastically-Coupled Composite Rotor Blades," $\mathrm{PhD}$ Dissertation from the University of Maryland, December 1993.

Nixon, M. W., Kvaternik, R. G., and Settle, T. B., "Tiltrotor Vibration Reduction Through Higher Harmonic Control," Proceedings of the CEAS International Forum on Aeroelasticity and Structural Dynamics, Rome, Italy, June 1997.

Scott, R. C. Wieseman, C. D., Hoadley, S. T., and Durham, M. H., "The Benchmark Active Controls Technology Model
Aerodynamic Data," Proceedings of the AIAA 35th Aerospace Sciences Meeting and Exhibit, Reno, NV, January 1997.

Silva, W., "Application of Nonlinear Systems Theory to Transonic Unsteady Aerodynamic Responses," AIAA Journal of Aircraft, Volume 30, Number 5, September-October 1993, pps 660668.

Silva, W., "Extension of Nonlinear Systems Theory to General-Frequency Unsteady Transonic Aerodynamic Responses," AIAA Paper No. 93-1590, Presented at the 34th Structures, Structural Dynamics, and Materials Conference, La Jolla, CA, April 19-21, 1993.

Staff of the Aeroelasticity Branch, "The Transonic Dynamics Tunnel," Langley Working Paper 799, July 1992.

Theodorsen, Theodore, "General Theory of Aerodynamic Instability and the Mechanism of Flutter," NACA Report 496, 1935.

Wilkie, W. K., Belvin, W. K., and Park, K. C., "Aeroelastic Analysis of Helicopter Rotor Blades Incorporating Anisotropic Piezoelectric Twist Actuation," AD-Vol. 52, Proceedings of the ASME Aerospace Division, November, 1996, pp. 423-433. 